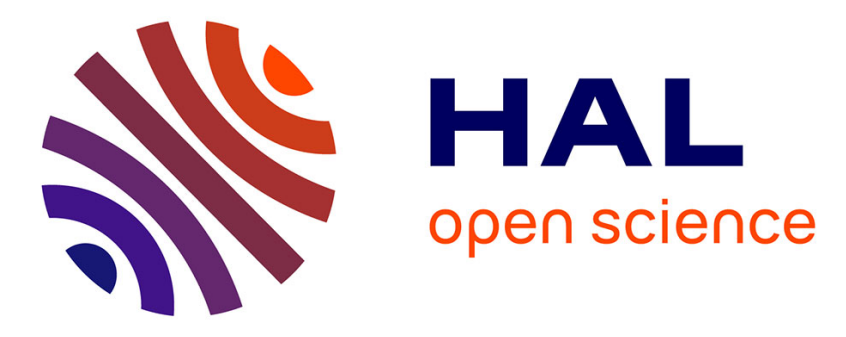

\title{
Human hippocampal neurons track moments in a sequence of events.
}

\author{
Leila Reddy, Benedikt Zoefel, Jessy Possel, Judith Peters, Doris Dijksterhuis, \\ Marlene Poncet, Elisabeth C.W. van Straaten, Johannes Baayen, Sander \\ Idema, Matthew Self
}

\section{To cite this version:}

Leila Reddy, Benedikt Zoefel, Jessy Possel, Judith Peters, Doris Dijksterhuis, et al.. Human hippocampal neurons track moments in a sequence of events.. Journal of Neuroscience, 2021, 41 (31), pp.6714-6725. 10.1523/JNEUROSCI.3157-20.2021 . hal-03087233v2

\section{HAL Id: hal-03087233 \\ https://hal.science/hal-03087233v2}

Submitted on 8 Nov 2021

HAL is a multi-disciplinary open access archive for the deposit and dissemination of scientific research documents, whether they are published or not. The documents may come from teaching and research institutions in France or abroad, or from public or private research centers.
L'archive ouverte pluridisciplinaire HAL, est destinée au dépôt et à la diffusion de documents scientifiques de niveau recherche, publiés ou non, émanant des établissements d'enseignement et de recherche français ou étrangers, des laboratoires publics ou privés. 


\section{Human hippocampal neurons track moments in a sequence of events.}

Abbreviated title: Time cells in the human hippocampus.

5 Leila Reddy ${ }^{1,2,3,}{ }^{*}$, Benedikt Zoefel ${ }^{1,2}$, Jessy K. Possel ${ }^{4,5}$, Judith C. Peters ${ }^{5}$, Doris Dijksterhuis ${ }^{4}$, Marlene Poncet ${ }^{1,2,6}$, Elisabeth C.W. van Straaten ${ }^{7}$, Johannes C. Baayen ${ }^{8}$, Sander Idema ${ }^{8}$, Matthew W. Self ${ }^{4}$

${ }^{1}$ Université de Toulouse, Centre de Recherche Cerveau et Cognition, Université Paul Sabatier, 31052, Toulouse, France.

${ }^{2}$ CNRS, UMR 5549, Faculté de Médecine de Purpan, 31052 Toulouse, France.

$10{ }^{3}$ Artificial and Natural Intelligence Toulouse Institute (ANITI), 31052 Toulouse, France.

${ }^{4}$ Vision and Cognition group, Netherlands Institute for Neuroscience, 1105BA, Amsterdam, The Netherlands

${ }^{5}$ Cognitive Neuroscience Department, Faculty of Psychology and Neuroscience, Maastricht University, Oxfordlaan 55, 6229 EV,

Maastricht, the Netherlands.

${ }^{6}$ University of St. Andrews, School of Psychology and Neuroscience, UK

$15{ }^{7}$ Dept of Neurology and Clinical Neurophysiology, Amsterdam UMC, Vrije Universiteit Amsterdam, De Boelelaan 1085, 1081HV Amsterdam, the Netherlands

${ }^{8}$ Department of Neurosurgery, VU University Medical Center, De Boelelaan 1085, 1081HV Amsterdam, the Netherlands

*Corresponding author. (leila.reddy@cnrs.fr)

Number of pages: 25

Number of figures: 6

Number of words

Abstract: $176 / 250$

Significance Statement: 100/120

Introduction: 506/650

Discussion: $1317 / 1500$

Conflict of interest: The authors declare no conflict of interest.

30 Acknowledgements: This work was supported by grants from the French Agence Nationale de la Recherche (ANR-12-JSH2-0004-01 and ANR-18-CE37-0007-01), ANITI (Artificial and Natural Intelligence Toulouse Institute) Research Chair (ANR-19-PI3A-0004), the Fyssen foundation, and the Université Paul Sabatier, Toulouse, France (BQR, 2009 and Appel à Projets de Recherche Labellisés, 2013), to L.R. JP was supported by an NWO Onderzoekstalent grant awarded to 35 MWS. DD was supported by an NWA-Startimpuls grant awarded to MWS. We would like to thank Pieter Roelfsema for his support. 


\section{Human hippocampal neurons track moments in a sequence of events.}

Abstract:

An indispensable feature of episodic memory is our ability to temporally piece together different elements of an experience into a coherent memory. Hippocampal "time cells" neurons that represent temporal information - may play a critical role in this process. While

45 these cells have been repeatedly found in rodents, it is still unclear to what extent similar temporal selectivity exists in the human hippocampus. Here we show that temporal context modulates the firing activity of human hippocampal neurons during structured temporal experiences. We recorded neuronal activity in the human brain while patients of either sex learned predictable sequences of pictures. We report that human time cells fire at successive moments in this task. Furthermore, time cells also signaled inherently changing temporal contexts during empty 10-second gap periods between trials, while participants waited for the task to resume. Finally, population activity allowed for decoding temporal epoch identity, both during sequence learning and during the gap periods. These findings suggest that human hippocampal neurons could play an essential role in temporally organizing distinct moments of

55 an experience in episodic memory.

\section{Significance Statement:}

Episodic memory refers to our ability to remember the "what, where, and when" of a past 60 experience. Representing time is an important component of this form of memory. Here, we show that neurons in the human hippocampus represent temporal information. This temporal signature was observed both when participants were actively engaged in a memory task, as well as during 10s-long gaps when they were asked to wait before performing the task. Furthermore, the activity of the population of hippocampal cells allowed for decoding one

65 temporal epoch from another. These results suggest a robust representation of time in the human hippocampus.

\section{Introduction:}

70 Creating episodic memories requires linking together distinct events of an experience with temporal fidelity. The brain must represent the temporal flow and order of events, and glue them together in the correct sequential order. "Time cells" in the hippocampus and adjacent structures might play an essential role in this temporal organization of memory (Hasselmo, 2009; Eichenbaum, 2014; Howard et al., 2014). In rodents, time cells signal changing temporal contexts in a variety of paradigms (Manns et al., 2007; Pastalkova et al., 2008; MacDonald et al., 2011; Kraus et al., 2013; MacDonald et al., 2013; Kraus et al., 2015). They fire at successive moments of time during a fixed interval and the activity of the population of time cells covers the entire time interval (Pastalkova et al., 2008). More recently, another class of "ramping cells" in the lateral entorhinal cortex has been discovered. Ramping cells show slowly rising or 
decaying activity with time, over a range of time scales. Temporal epoch identity could be decoded from the firing activity of the population of cells (Tsao et al., 2018).

Temporal coding has also been observed in neuronal activity patterns in the human hippocampus. For instance, neuronal activity in the human medial temporal lobe shows gradual changes over time in memory tasks (Howard et al., 2012; Folkerts et al., 2018). The recall of a particular item is accompanied by the reinstatement of its initial temporal representation (Gelbard-Sagiv et al., 2008; Howard et al., 2012; Folkerts et al., 2018). More recently, single neurons have also been shown to be modulated by time, akin to time cells in rodents, during encoding and retrieval in a free recall memory task (Umbach et al., 2020).

In the current study, we ask if human hippocampal neurons represent temporal information during sequential order learning. A large body of work in animals and humans has shown that the hippocampus is essential for remembering the temporal order of sequential events (Eichenbaum, 2013). For example, in humans, the hippocampus is activated when subjects 95 recall the order of objects, and conversely, patients with hippocampal damage have trouble in temporal order judgements (Spiers et al., 2001; Ekstrom and Bookheimer, 2007). In animals, rats with hippocampal damage are impaired at remembering the sequential order of odors (Fortin et al., 2002). Given the importance of the hippocampus in sequence order learning and temporal order judgements, we tested whether human hippocampal neurons represented 100 temporal information while participants learned the order of a sequence of items. We tested for temporal modulation of hippocampal activity in two experiments: (1) during sequence learning (Figure $1 \mathrm{~A}$ ), and (2) during empty gap periods inserted in the task during which participants passively waited for the sequence to resume (Figure 1B). Note that in these gap periods, any potential temporal information is not driven by external stimuli or events, but 105 rather represents inherent changes in the patients' moment-to-moment experience. We report that human hippocampal neurons fire at successive moments during these structured time periods, both while subjects actively monitor a sequence, as well as during empty temporal gaps between events.

A Experiment 1: Sequence Learning

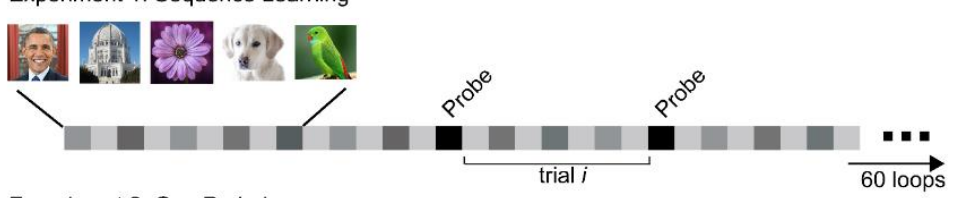

B

Experiment 2: Gap Periods

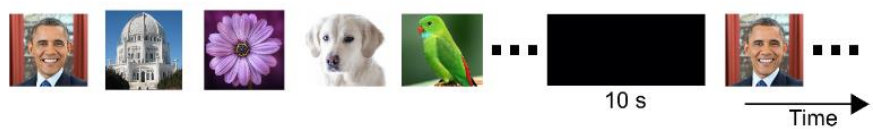

Figure 1. Experimental Design. A). In the sequence learning experiments, participants saw a sequence of images in a fixed order, and were asked to learn the sequence order. The stimulus sequence consisted of 5-7 image periods (image number fixed per session and determined by the availability of the patient) separated by inter-stimulus 115 interval (ISI) periods. Each image was presented for $1.5 \mathrm{~s}$ followed by an ISI of $0.5 \mathrm{~s}$. The sequence was repeated for 60 loops. 20\% of the time, a probe event occurred (black squares) during which participants had to decide which of two choice images was the correct one at the current position in the sequence. The probe events occurred at 
random positions of the sequence. After the probe event, the sequence resumed. In our main analysis, we consider time periods that occurred between two consecutive probe events as the "trials" of interest. As shown, each post-probe "trial" consisted of several image and ISI periods (gray squares). B) The design of Experiment 2 was similar to that of Experiment 1 except for the insertion of 10-second-long gap periods (black rectangle) during sequence learning. These gap periods occurred periodically (see Methods). During the gap periods, the sequence stopped and patients were presented with a blank screen. They were asked to simply wait until the sequence resumed.

\section{Materials and Methods:}

In this study, human epileptic patients performed two sequence learning tasks, while single neuron activity was recorded from microelectrodes implanted in the hippocampus (Figure 2).

130 We quantified the influence of time on the firing activity of individual neurons using a stepwise general linear model (GLM), as has previously been used in the rodent literature (MacDonald et al., 2011; Tsao et al., 2018). In this GLM, a predictor variable is included in the model only if it is found to significantly improve the prediction of the response variable (see below).

\section{Patients}

Nine patients of either sex participated in the first experiment, and six patients of either sex participated in the second experiment. The patients were diagnosed with pharmacologically intractable epilepsy, and were undergoing epileptological evaluation at the Amsterdam

140 University Medical Center, location VUmc, The Netherlands. Patients were implanted with chronic depth electrodes for 7-10 days in order to localize the seizure focus for possible surgical resection (Fried et al., 1997; Engel et al., 2005). All surgeries were performed by J.C.B and S.I. The Medical Ethics Committee at the VU Medical Center approved the studies. The electrode locations were based entirely on clinical criteria and were evaluated based on the pre-surgical 145 planned trajectories on the basis of structural MRI scans. For each electrode the planned trajectory was adjusted to ensure that the tip of the macroelectrode was at least $3 \mathrm{~mm}$ within the body of the hippocampus. The clinical team aimed for microwires that extended approximately $2-3 \mathrm{~mm}$ from the tip of the macroelectrode. The accuracy of the implantation was always checked using a CT scan co-registered to the MRI. We only included electrodes that

150 were within a $3 \mathrm{~mm}$ deviation from the target (based on visual confirmation). Each electrode contained eight microwires (Behnke-Fried electrodes, Ad-Tech Medical) from which we recorded multi-unit activity, and a ninth microwire that served as a local reference. The signal from the microwires was recorded using a 64-channel Neuralynx system, filtered between 1 and $9000 \mathrm{~Hz}$, sampled at $32 \mathrm{KHz}$. On average, each patient was implanted with $34 \pm 11.8$ 155 microwires (range $=[16,48]$ ). Participants sat in their hospital room at the Epilepsy Monitoring Unit, and performed the experimental sessions on a laptop computer.

\section{Spike Detection and Sorting}

160 Spike detection and sorting were performed with wave_clus (Quiroga et al., 2004). Briefly (see (Reddy et al., 2015) for details), the data were band pass filtered between $300-3000 \mathrm{~Hz}$ and spikes were detected with an automatic amplitude threshold. Spike sorting was performed with 
a wavelet transform that extracted the relevant features of the spike waveform. Clustering was performed using a super-paramagnetic clustering algorithm. Clusters were visually reviewed by the first-author for 1 ) the mean spike shape and its variance; 2) the ratio between the spike peak value and the noise level; 3) the inter spike interval distribution of each cluster; 4) the presence of a refractory period; 5 ) the similarity of each cluster to other clusters from the same microwire. Based on manual inspection of these criteria, clusters were retained, merged or discarded.

170

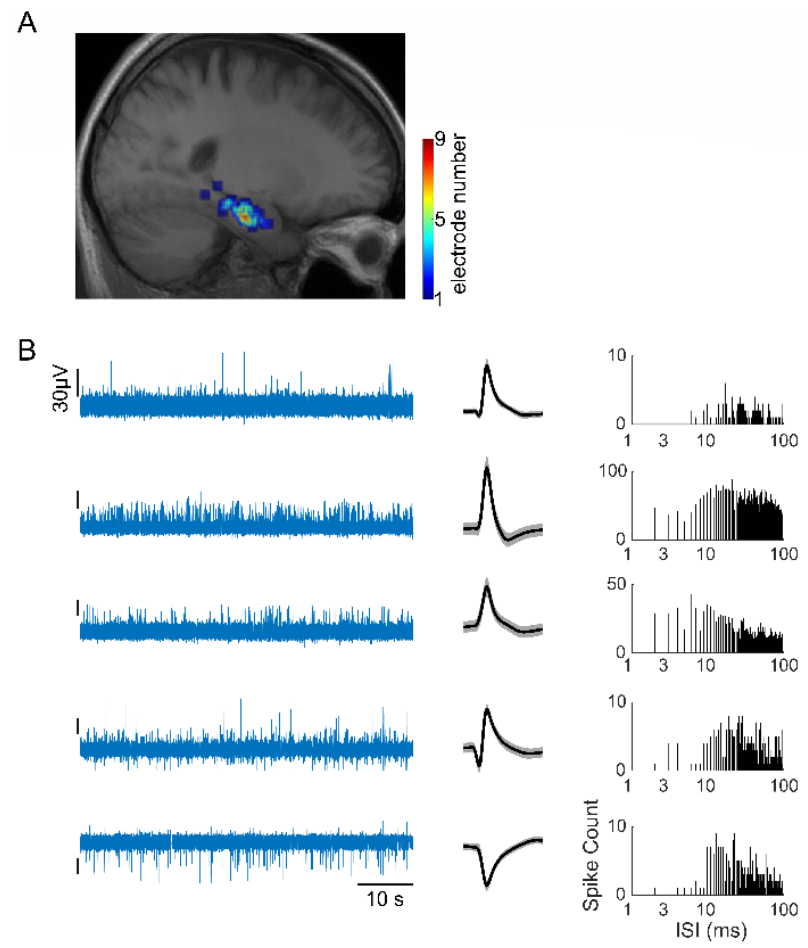

Figure 2. A). Electrode locations shown on a sagittal slice of the average MRI of patients registered to the MNI brain template. B). Band-pass filtered $(300-3000 \mathrm{~Hz})$ signal from five different channels (left), mean waveforms recorded on these channels (middle), and the corresponding distributions of inter-spike intervals (right). The black vertical tick marks on the left plots indicate a scale of $30 \mathrm{uV}$.

\section{Experimental design and statistical analyses}

\section{Behavioral Task}

Experiment 1: Sequence Learning: The patients performed a total of 31 sequence learning (SL) sessions (Figure 1A). In each SL session, participants were presented with a sequence of 5-7 images (image number determined as a function of the difficulty level and the availability of the patient). The images were always presented in a pre-determined order such that a given image, 185 A, predicted the identity of the next image, B, and so on. Subjects were asked to remember the order of the images in the sequence. Each image was presented for 1.5s ("image period") followed by an "inter-stimulus interval period" (ISI) of $0.5 \mathrm{~s}$. The sequence was repeated continually 60 times resulting in experimental sessions of 10 minutes for 5 -image sequences and 14 minutes for 7-image sequences, not including time spent by the subject to respond on 
190 probe events. On a random $20 \%$ of image periods, the sequence stopped and participants were presented with probe events. In these probe events, instead of being presented with the next image of the sequence, subjects were shown two images side by side and asked to decide (by pressing one of two keys on the keyboard) which of the two was the next image in the sequence. After the subjects had responded, the sequence resumed.

195 From the point of view of the subject, the probe events were salient moments of an otherwise repetitive experiment because the probes stopped the sequence and tested subjects on their learning of the sequence order. Thus, we considered sequence segments between two consecutive probe events as our "trials" of interest: structured, temporal experiences between two salient markers. We asked whether hippocampal neurons tracked time in this interval. In

200 control analyses described below, we verified these results with respect to other time periods in the experiment.

Experiment 2: Sequence learning with temporal gaps (Figure 1B): Six new patients performed eight sessions of a second SL experiment. This second experiment followed the design of the 205 first SL experiment described above, except for the following modifications: 1) After a fixed number of repeats of the sequence, a 10s-long empty gap interval was presented. During these gap intervals, participants were presented with a black screen, without any stimulus input. They were asked to simply wait until the sequence started again. For three participants these gap intervals occurred after every 5 repeats of the sequence (resulting in 6 gap intervals in the

210 experiment), while for the remaining three participants these gap intervals occurred after every 2 repeats of the sequence (resulting in 15 gap intervals in the experiment). 2) The sequence was repeated only 30 times instead of 60 times.

In the nine patients who performed the first experiment, we recorded from 429 neurons in the

215 hippocampus, and in the six patients who performed the second experiment, we recorded 96 hippocampal units.

\section{Time Cell Identification with a General Linear Model (Experiment 1)}

220 Time cell identification was performed with a GLM as in previous studies (MacDonald et al., 2011; Tsao et al., 2018). The firing activity of each neuron was modelled as a function of time, image identity, and whether the temporal period corresponded to an image or ISI period.

For the purposes of the GLM, as described above (Figure 1A), we defined "trials" as segments of 225 the sequence between two consecutive probe events (number of sequence segments or "trials" between two consecutive probe events across sessions: mean \pm s.e.m=73.6 \pm 2.4 ). We made this choice because (i) as explained previously, these probe events were the most salient events of the experiment, and (ii) if we simply consider time $=0$ as the start of each 5-7 image sequence, "time" in the sequence is directly confounded by image identity because the sequence order is

230 fixed. By redefining time $=0$ as the time at which the sequence restarted after the probe events, we avoided this confound because time is not confounded with image identity with respect to probe events (the sequence segment after the probe is random since the probe events 
occurred at random moments). In control analyses we also considered different temporal intervals for determining time cells.

Each of the post-probe "trials" consisted of several image and ISI periods that regularly followed each other (Figure 1A). The median number of image and ISI periods in a "trial" was seven, corresponding to a median trial length of 6.5 seconds. For all subsequent analyses, only the first $6.5 \mathrm{~s}$ of each trial were included in the GLM (i.e., trials shorter than this duration were 240 excluded from the GLM). Thus, each "trial" between probe events was a well-structured temporal interval during which the sequence progressed according to its fixed order. The average number of trials included in the GLM was $49.7 \pm 0.8$ (mean \pm standard deviation, range $=[25,64])$.

245 In the GLM, the firing activity vector $(Y)$ on each "trial" contained the average firing rates for each period of the trial, with no smoothing or additional preprocessing. $Y$ was modelled as a function of three variables: image identity in each period, time of the period (i.e., time of the mid-point of the period with respect to trial onset/probe offset; time varied between [0 6.5s]), and whether the period corresponded to an image or an ISI event. A linear factor for time 250 assumes that time cells either show a ramping up or a ramping down of firing activity during the trial. To also include the possibility of cells having a preferred time not just at the beginning and end of trials, but also at intermediate points, we included a quadratic time term (i.e., $\mathrm{t}^{2}$, a parabola-shaped function; for this purpose, time was re-centered to the middle of the trial and thus varied between [-3.25 3.25s]). Note that a linear combination of a linear and quadratic 255 term allows for detecting maximal/minimal firing at any point of the time window of interest.

$$
Y=\beta_{0}+\beta_{1} \text { ImageOrISI }+\sum_{i} \beta_{i} \text { ImageID }_{i}+\beta_{j} \text { Time }+\beta_{k} \text { Time }^{2}(1)
$$

The GLM analysis was performed using the MATLAB stepwiselm function, including the 260 variables image/ISI, imageID, Time and Time $^{2}$, in a linear model, with a constant term as the baseline model, the SSE criterion (PEnter $=0.05)$, and other default parameters. The variables image/ISI and imageID were entered as categorical variables, and the time variables were continuous variables. Stepwise regression systematically tests the variance explained by adding and removing variables from a linear model based on their statistical significance in explaining 265 the response variable. Note that the order in which regressors are entered into the stepwise linear model does not affect its outcome. Time cells were defined as cells for which the time terms (i.e., Time and/or Time ${ }^{2}$ ) were added by the stepwiselm function (PEnter $<0.05$ ).

Statistical testing was performed for each time cell with permutation testing in which the firing 270 rates were shuffled with respect to the task design, and the stepwise regression repeated. This procedure was repeated 1000 times per cell. For each cell, $p_{\text {actual }}$ was the $p$-value returned by the regression analysis for the real data. $p_{\text {actual }}$ was compared to the distribution of $p$-values returned from the shuffling procedure. For cells where both the time and time $^{2}$ terms were significant the $p$ value corresponded to the smaller of the corresponding $p$-values. We defined

$275 p_{\text {shuffle }}$ as the proportion of shuffles that produced a smaller $p$ value than $p_{\text {actual. }}$. Only cells with $p_{\text {shuffle }}<0.05$ were ultimately considered time cells in this analysis (Umbach et al., 2020). 
As a separate test to confirm our classification of time cells, different from the stepwise regression test, we performed a likelihood ratio test to compare the log-likelihood values of a restricted linear model which included all terms except the time terms, and a full model which also included the two time terms.

\section{Time Cell Identification with a General Linear Model (Experiment 2)}

285 The 10s gap intervals of experiment 2 were epoched into 500ms non-overlapping windows and, as above, a stepwise regression analysis was performed: the firing activity $(Y)$ in each epoch was modelled as a function of time in the epoch (Time and a quadratic time term Time ${ }^{2}$ ). All other parameters in this analysis were identical to those described for Experiment 1 . Statistical testing was performed for each time cell with the permutation method described above.

$$
Y=\beta_{0}+\beta_{1} \text { Time }+\beta_{2} \text { Time }^{2}(2)
$$

\section{Control analyses for defining trial periods and time cells}

In the main analysis of Experiment 1, a "trial" was defined with respect to the probe events (i.e., the sequence segment that occurred between two consecutive probe events). We performed several additional analyses for identifying time cells; in each case permutation testing was performed for each time cell as described above. The results of the control analyses are summarized in Table 1.

1) Time cells were identified when the first period after the probe event was excluded from the GLM. 2) Time cells were identified when the GLM analysis was performed on only the ISI periods. In this control, the $Y$ vector contained the firing rates in the ISI periods, and the

305 regressor matrix $X$ consisted of the time factors and an image identity factor (i.e., the identity of the image following the ISI period, to account for image-specific anticipatory responses that can be observed in the ISI periods during sequence learning (Reddy et al., 2015)). 3) "Trials" were re-defined as sequence segments with respect to the onset of each repetition of the sequence. Note that in this case, time selectivity can be confounded by image selectivity (since 310 the same stimulus sequence repeats identically in every loop); however, the Matlab stepwiselm function that we used for determining time selectivity could disentangle the potential contributions of the time and image ID variables since it systematically tests for the addition and removal of each variable in significantly explaining the response variable. Nonetheless, to avoid any ambiguity in interpretation, we elected to present time selectivity with respect to 315 probe events as our main analysis, as it precludes this potential confound. 4) Time cells were also identified in a control analysis that used an N-way ANOVA to test for an interaction between firing rates and time, as in Umbach et al., 2020. For each trial we computed the firing rate within each ISI and image period. We tested for temporal modulation of firing activity with predictors time bin, image identity and image/ISI. As in Umbach et al., 2020, cells with a significant main effect of time (i.e., $p_{\text {actual }}<0.05$ ) from this procedure were passed on for 
statistical testing. Statistical testing was performed with permutation testing in which the firing rates were shuffled with respect to the task design, and the ANOVA was repeated on the shuffled data. This procedure was repeated 1000 times for each cell. $p_{\text {actual }}$ was compared with the distribution of $p$-values obtained from the shuffling procedure. We defined $p_{\text {shuffle }}$ as the proportion of shuffles that produced a smaller $p$ value than $p_{\text {actual. }}$ Only potential time cells with $p_{\text {shuffle }}<0.05$ were ultimately considered time cells in this analysis. 5) To identify ramping cells (Tsao et al., 2018), we repeated the stepwise GLM approach as in the main analysis but with the exclusion of the quadratic time term. A summary of the control analyses is found in Table 1.

\begin{tabular}{|c|c|c|}
\hline Control Analysis & $\begin{array}{l}\text { Number of time cells } \\
\text { identified (\% overlap with } \\
\text { time cells from the main } \\
\text { analysis) }\end{array}$ & Concern \\
\hline Likelihood ratio test & $138(84.7 \%)$ & $\begin{array}{l}\text { Identification of time cells is } \\
\text { method-dependent. }\end{array}$ \\
\hline $\begin{array}{l}\text { Stepwise GLM on ISI } \\
\text { periods only }\end{array}$ & $106(76.4 \%)$ & $\begin{array}{l}\text { Identification of time cells is } \\
\text { dependent on image information. }\end{array}$ \\
\hline $\begin{array}{l}\text { Stepwise GLM excluding } \\
1^{\text {st } I S I}\end{array}$ & $65(81.5 \%)$ & $\begin{array}{l}\text { Identification of time cells is } \\
\text { dependent on the probe events. }\end{array}$ \\
\hline $\begin{array}{l}\text { Stepwise GLM w.r.t } \\
\text { sequence onset }\end{array}$ & $69(30.4 \%)$ & $\begin{array}{l}\text { Identification of time cells is } \\
\text { dependent on the temporal } \\
\text { interval. }\end{array}$ \\
\hline N-way ANOVA & $101(88.1 \%)$ & $\begin{array}{l}\text { Identification of time cells is } \\
\text { method-dependent. }\end{array}$ \\
\hline $\begin{array}{l}\text { Stepwise GLM excluding } \\
\text { the quadratic time term }\end{array}$ & $114(88.7 \%)$ & Identification of "ramping" cells. \\
\hline
\end{tabular}

Table 1: The number of time cells identified in the control analyses and the overlap between time cells identified in the main analysis and the control analyses.

\section{Statistical test for the number of time cells}

Statistical significance for the number of time cells identified by the GLM analysis was evaluated using a permutation test. For the permutation test, in Experiment 1, surrogate data was created by randomly shuffling the image and ISI periods on each trial. For Experiment 2 surrogate data was created by randomly shuffling epoch time. The stepwise regression was then performed on this surrogate data, on $10^{6}$ iterations. The proportion of surrogates which had a higher number of time cells was $p<10^{-6}$ in all analyses (in other words, none of the surrogates ever had a higher number of time cells).

Heatmaps cross-validation and statistical test 
The "original" heatmaps shown in Figures 3B and 5B were constructed by sorting the time cells 345 according to the latency of peak firing and using this sorted order to plot the firing rate of each cell over the time interval. However, a heatmap generated from random data, sorted and plotted according to the maximum value of each entry, will also show a similar well-organized pattern. Thus, to ensure the reliability of these maps, we performed a cross-validation analysis on the real heatmaps to verify their reproducibility.

350 For the cross-validated heatmaps, the order in which the cells were plotted was determined from the latencies on a random half of the data and the firing rates were plotted for the remaining half of the data. If the cells had no true temporal preference, the peak latency on the second half should be unrelated to the latency measured on the first half, and no meaningful ordering of cells should appear. This cross-validation procedure was repeated 1000 times, and

355 the resulting heat maps were averaged across cross-validations to generate cross-validated maps. We quantified the reliability of the heatmaps by performing a non-parametric permutation test. We first computed the Spearman correlation point-wise between the average cross-validated heatmap and the original heatmap, resulting in a correlation measure

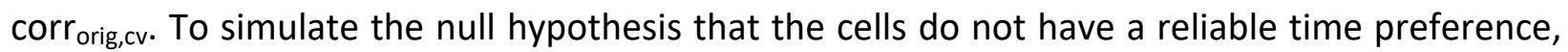
360 on each cross-validation iteration we generated $10^{5}$ surrogate heatmaps by randomizing the cell order obtained from the first half of the data (instead of determining their order based on peak firing time over the first half), and generating a heatmap with this random order for the second half of the data. Each of these $10^{5}$ surrogate heatmaps was correlated with the original

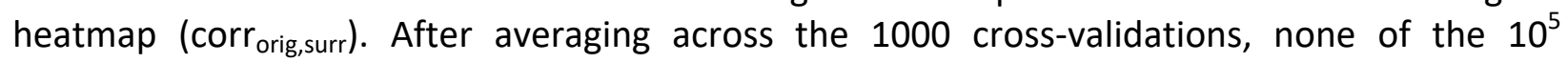

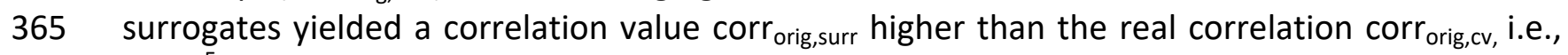
$p<10^{-5}$.

\section{Population pattern analysis}

370 For this analysis, the population size was 429 neurons for Experiment 1, and 96 neurons for Experiment2.

Experiment 1: Sequence learning: The population pattern analysis for Experiment 1 was performed on the image periods. The population pattern analysis was based on a pairwise 375 comparison of image periods, thus chance performance for all analyses described below is $50 \%$.

As mentioned above, each "trial" (i.e., the sequence segment between two consecutive probe events) consisted of a varying number of image periods (Figure $1 \mathrm{~A}$ ). In the population pattern analysis our goal was to discriminate temporal period identity (e.g., image period 1 vs. image 380 period 2 etc.). Pairwise discrimination performance was evaluated for different numbers of temporal periods from the start of the trial (ranging from two to five; i.e., discriminating between the first two image periods after the probe event, the first three image periods and so on). To ensure that performance was not driven by an unequal representation of the different images in the different periods, we created a balanced dataset for the population pattern 385 analysis. In this balanced dataset we included the subset of trials per cell that assured that each image was equally present in each period (Figure 6A). The balanced dataset only included cells 
for which a minimum number of trials had been recorded. The minimum number of trials was the smallest number such that at least 300 cells were included in the analysis.

390 For the 2-way discrimination (period 1 vs. period 2), the balanced dataset required 42 trials, and 397 cells were included; for a 3-way discrimination (period 1 vs. period 2 vs. period 3) it required 30 trials, and included 303 cells; for a 4-way discrimination it required 12 trials and included 320 cells; and for a 5-way discrimination, it required 6 trials and included 367 cells. Discrimination of temporal period identity in this balanced dataset was thus not influenced by

395 an imbalance in the number of presentations of each image across temporal periods. Note that the balanced dataset did not include the first ISI period after the probe and decoding was thus not influenced by the offset of the probe events. As mentioned below, trial selection for creating the balanced dataset was repeated on 50 iterations, and the population pattern analysis was performed on each iteration.

400

The population pattern analysis was performed using a split-half approach (Haxby et al., 2011; Pereira et al., 2018) on the firing rates of the balanced dataset (Figure 6B-D). The trials in the balanced dataset were randomly split into two halves, and in each half the firing activity of the population of neurons was arranged into vectors, per period and per trial (Figure 6B). These 405 vectors were averaged across trials, yielding a population pattern vector for each period ("period vectors") in each half of the dataset (Figure 6C). To quantify decoding or discrimination performance, we pairwise correlated the period vectors in one half of the dataset with the period vectors in the other half, and used the pairwise correlation values to measure the percentage of correct classification (Figure 6D). To be more precise, for each period we 410 computed the correlation for this period across the two halves of the dataset ("within" comparison, $\left.r_{\text {within }}\right)$, and compared it to the correlation with a different period in the other half of the dataset ("between" comparison, $r_{\text {between }}$ ). Decoding was correct if the "within" comparison was larger than the "between" comparison (Figure 6G), (Haxby et al., 2001). This procedure was repeated for all pairs of periods, and pairwise decoding accuracy was the 415 proportion of correct comparisons. Since this procedure was based on pairwise comparisons, chance performance was $50 \%$ for all analyses. Feature normalization was performed on the dataset for this analysis by performing a z-score on the data for each cell along the periods dimension. Feature normalization was performed on the whole dataset based on the mean and standard deviation measured in the "training" half of the dataset (to avoid "leakage" of 420 information from the training half to the test half during the split-half cross-validation).

Note that in the split-half approach the "training data" (one half of the data) and "testing data" (the other half of the data) are independent by construction.

425 To increase reliability, the population pattern analysis was performed over several iterations: (i) trial selection for the balanced dataset was repeated 50 times; (ii) on each of these 50 iterations, the dataset was randomly split into two halves 200 times. The reported mean decoding accuracy was the average decoding performance across the 50 iterations for creating the balanced dataset. The standard deviation was computed across the 50 balanced datasets of 430 the mean decoding accuracy across the 200 split halves. Statistical significance was computed 
with a t-test against chance (0.5) across the 50 iterations for creating the balanced dataset (all $p$ $\left.<10^{-3}\right)$.

To compare decoding performance for time cells and non-time cells ("other cells"), and to determine how population size affects decoding accuracy, we performed the pairwise pattern analysis separately for time cells and other cells, and for different population sizes. Sizematched populations were generated by randomly subsampling the population of cells. For each population size, the same procedure described above was repeated on 20 balanced datasets and 50 split halves.

Experiment 2: Sequence learning with temporal gaps: For the population pattern analysis of the gap intervals, each gap interval was split into four, five or ten discrete periods and discrimination of temporal period identity was performed using a split-half approach (50 iterations). The procedure was the same as the one described above for Experiment 1 (Figure

445 6), and as above, chance performance was $50 \%$ since the method was based on pairwise comparisons. Feature normalization was performed on the dataset by performing a z-score on the data. Statistical significance was tested using a t-test against chance performance (0.5) across the 50 split-half iterations. Since some subjects had 6 gap periods while others had 15 gap periods, on each of the 50 iterations for splitting the data a random set of 6 gap periods

450 was chosen from the datasets which contained 15 gap periods.

\section{Results:}

\section{Behavioral task and number of units}

To determine whether human hippocampal neurons are modulated by temporal context, we recorded from hippocampal neurons in human patients who performed a sequence learning task (Figure $1 \mathrm{~A}, \mathrm{~B}$ ). We performed two independent sequence learning experiments in two groups of patients implanted with intracranial micro-electrodes. In Experiment 1, we recorded

460 the activity of 429 hippocampal neurons in nine patients, and in Experiment 2 we recorded from 96 hippocampal neurons in a new group of six patients.

In both experiments, the patients were presented with a fixed number of images (5-7, depending on the patients' availability) in a pre-defined order (Figure $1 \mathrm{~A}, \mathrm{~B}$ ), and asked to learn 465 the sequence order (Reddy et al., 2015). The sequence was repeated continually 60 times. Experiment 2 was similar to Experiment 1 except for the periodic insertion of 10-second-long gap periods during the experiment. During these gap periods, the sequence stopped and the patients had to wait until the sequence resumed (Figure 1B).

470 The duration of each image period in the sequence was $1.5 \mathrm{~s}$, and each image period was followed by an inter-stimulus interval (ISI) of $0.5 \mathrm{~s}$. On a random $20 \%$ of image periods, subjects were probed on their learning of the image order. During these probe events, the sequence momentarily stopped and subjects were presented with two images from the sequence. Their task was to report which of the two images was the correct one at the current sequence 
475 position. The sequence then resumed until the next probe event. Participants rapidly learned the sequence order and achieved $>90 \%$ performance on probe trials within the first six sequence presentations (Reddy et al., 2015). From the point of view of the subjects the probe events were salient moments of the experiment because they stopped the ongoing sequence and tested learning.

\section{Human hippocampal neurons are modulated by time during sequence learning}

Time cells have been characterized as neurons whose activity is modulated by temporal context within a well-defined time window. Our experiment design lent itself to identifying time cells

485 because the task consisted of a structured image sequence that occurred in a fixed and predictable time interval. In the time domain, our experiment involved three distinct timelines: (1) experiment time running from the beginning to the end of the experiment, (2) sequence time with respect to the start of each iteration of the sequence, and (3) probe time running between consecutive probe events. We first focus on probe time since, as described above, the 490 probe events were salient moments from the point of view of the participants. Furthermore, focusing on probe time allowed us to decouple time from image identity, since the post-probe trials consisted of varying segments of image and ISI periods.

We defined "trials" as segments of the experiment that occurred between two consecutive 495 probe events in Experiment 1 (Figure 1A). These trials were of a relatively fixed duration (median trial length $=6.5$ seconds), and consisted of a sequence of image and ISI periods. To identify time cells, we examined whether the firing activity of hippocampal neurons was modulated by time. Previous studies have identified time cells using a variety of frameworks such as fitting a Gaussian function to firing activity (Park et al., 2014; Salz et al., 2016), a one500 way ANOVA of time and firing rate (Umbach et al., 2020), or a general linear model (GLM) factoring in the influence of time and other experimental factors on firing activity (MacDonald et al., 2011; Tsao et al., 2018). In the current study we elected to use the stepwise general linear model (GLM) method established by Tsao and colleagues (Tsao et al., 2018), since it allows us to identify time cells while also measuring the influence of the other experimental 505 parameters on hippocampal responses.

In the stepwise GLM framework used here, a predictor variable is included in the model only if it is found to significantly improve the prediction of the response variable (firing rate). For each neuron, we modelled the firing rate in each image/ISI period of the trial sequence with different 510 potential predictors: image identity, period type (i.e., image or ISI period), and two time terms (a linear and a quadratic time term). A linear combination of the linear and quadratic terms allowed for detecting maximal/minimal firing at all points in the time window of interest. Time cells were identified as those in which one or both of the time terms was selected for inclusion in the stepwise linear model. To determine how likely it is that the temporal modulation of

515 firing rates exhibited by each time cell arose due to chance, we performed a shuffling procedure. Separately, statistical significance for the number of time cells was evaluated using a permutation test in which the image and ISI periods on each trial were randomly shuffled on $10^{6}$ iterations, and the stepwise GLM was performed on each iteration (Methods). 
520 We identified a significant number of hippocampal neurons (30\%) that were modulated by time during sequence learning (128 of 429 neurons passed the shuffling test, and this number was more than expected by chance based on a permutation test, $p<10^{-6} ; 106$ of these neurons were located in the anterior hippocampus and 22 in the posterior hippocampus). Individual examples of time cells are shown in Figure 3A. The reliability of time cells was evaluated by comparing the 525 regressor values for the time variables on odd/even trials, or the first half/second half of trials (Figure 4A, B). 107 time cells were modulated by the linear time term, 62 by the quadratic time term, and 41 by both time terms. Of the linearly modulated cells, $32.7 \%$ decreased their firing rates and $67.3 \%$ increased their firing rates (Figure 4F). To test for the presence of "ramping cells" (Tsao et al., 2018), we reran the analysis with a model that excluded the quadratic time term. In this analysis, $27 \%$ of hippocampal neurons (114/429) were significantly modulated by the linear time term, with a $94 \%$ overlap with the linearly modulated cells identified by the full model. This proportion of ramping cells is similar to a previous report by Umbach and colleagues, who reported $34.5 \%$ of ramping cells in the human hippocampus (Umbach et al., 2020).

A

Sequence Learning
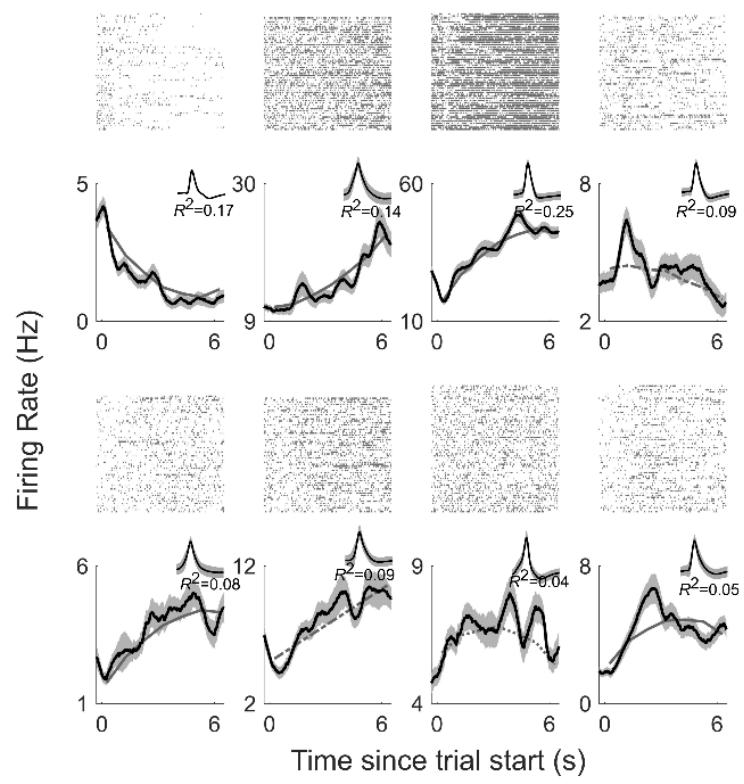

B

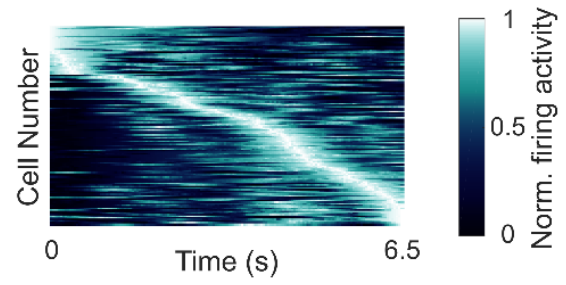


Figure 3. A) Hippocampal neurons are modulated by time during sequence learning. Raster plots (top) and poststimulus time histograms (bottom) are shown for 8 example time cells. The $x$-axis corresponds to time of the median trial length (6.5s, see Methods). The black line is the average firing activity and the shaded area corresponds to the standard error of the mean across trials. The gray lines show the model fit (solid gray line for cells that were classified as time cells according to the inclusion of the linear and quadratic terms, dashed gray line for cells classified based on the linear term alone, and stippled gray line for cells classified based on the quadratic term alone). The insets show the waveforms and the $R^{2}$ of the model for each cell. B) Hippocampal neurons fire at successive moments of a temporal interval. Firing activity of the population of time cells $(N=128)$ identified as being significantly modulated by time (i.e., 'time' and/or 'time ${ }^{2 \text { ) }}$ in the sequence learning experiment. Each row shows the firing activity for an individual time cell, averaged across trials. The $x$-axis corresponds to time of the median trial length. The neurons are sorted by the latency of the maximum firing rate.

550

In a separate analysis to confirm our classification of neurons as time cells, we performed a likelihood ratio test to compare the log-likelihood values of a restricted linear model which included all terms except the time terms, and a full model which also included the time terms. Time cells were identified as cells which had a significantly higher log-likelihood value according 555 to the likelihood ratio test ( $p<0.05 ; 138$ cells identified). $85 \%$ of cells based on this method overlapped with the population identified by the stepwise regression method (Table 1 ).

We conducted a number of control analyses to verify that the temporal modulation of firing rates was observed under different analysis parameters (Table 1 and Figure 4D). Time cells 560 were identified when the GLM was performed on the firing rates of only the ISI periods (106 cells, permutation test, $p<10^{-6}$ ), and when excluding the first ISI period after the probe (65 cells, permutation test, $\left.p<10^{-6}\right)$. Time cells were also detected when "trials" were re-defined as sequence segments with respect to the onset of each repetition of the sequence (i.e., instead of with respect to the onset of the probe events, as in the main analysis). In this analysis, we

565 identified 69 neurons that were significantly modulated by the time variables (permutation test, $\left.p<10^{-6}\right)$. Finally, time cells were also detected based on an ANOVA (101 cells) as in (Umbach et al., 2020).

Thus, human hippocampal neurons represent a changing temporal context while participants 570 are actively engaged in memorizing the order of a sequence of events. Previous studies have shown that when considered at the population level, the firing activity of time cells covers the duration of a given temporal epoch (Pastalkova et al., 2008; MacDonald et al., 2011). Likewise, across all participants we observed neuronal peak firing at successive moments in time, and when each cell was ordered by its preferred moment of firing, population activity spanned the 575 temporal window (Figure 3B). For consistency with previous studies, we illustrate this data as a population level heatmap. Although population level heatmaps are primarily used for display purposes, it can be informative to test their reliability, since random data sorted by peak value could also generate well-organized heat maps. To test the reliability of our heat maps we performed a cross-validation analysis, in which the preferred time of firing for each cell was 580 determined in one half of the data and the consistency of time preference was measured in the second half of the data. Statistical significance of the cross-validated heatmaps was evaluated using a permutation method (Methods), in which cross-validated and surrogate (randomly permuted) heatmaps were correlated with the original heatmap. The proportion of surrogates 
which had a higher correlation than the cross-validated data was $p<10^{-5}$, supporting the notion that temporal preference was reliable in our neuronal population.

An advantage of the GLM-based approach used in the present study is that it allowed us to tease apart the influence of different experimental factors on the firing activity of hippocampal neurons (Figure 4C, E, G, H). The GLM analysis quantified the influence of stimulus presence 590 (i.e., image periods vs. ISI periods), image identity, and time in the trial. A considerable number of neurons was modulated exclusively by time ( 111 cells) or image identity ( 50 cells), but we also found neurons selective for a combination of these factors (17 neurons for time and another factor; 17 neurons for image identity and another factor).
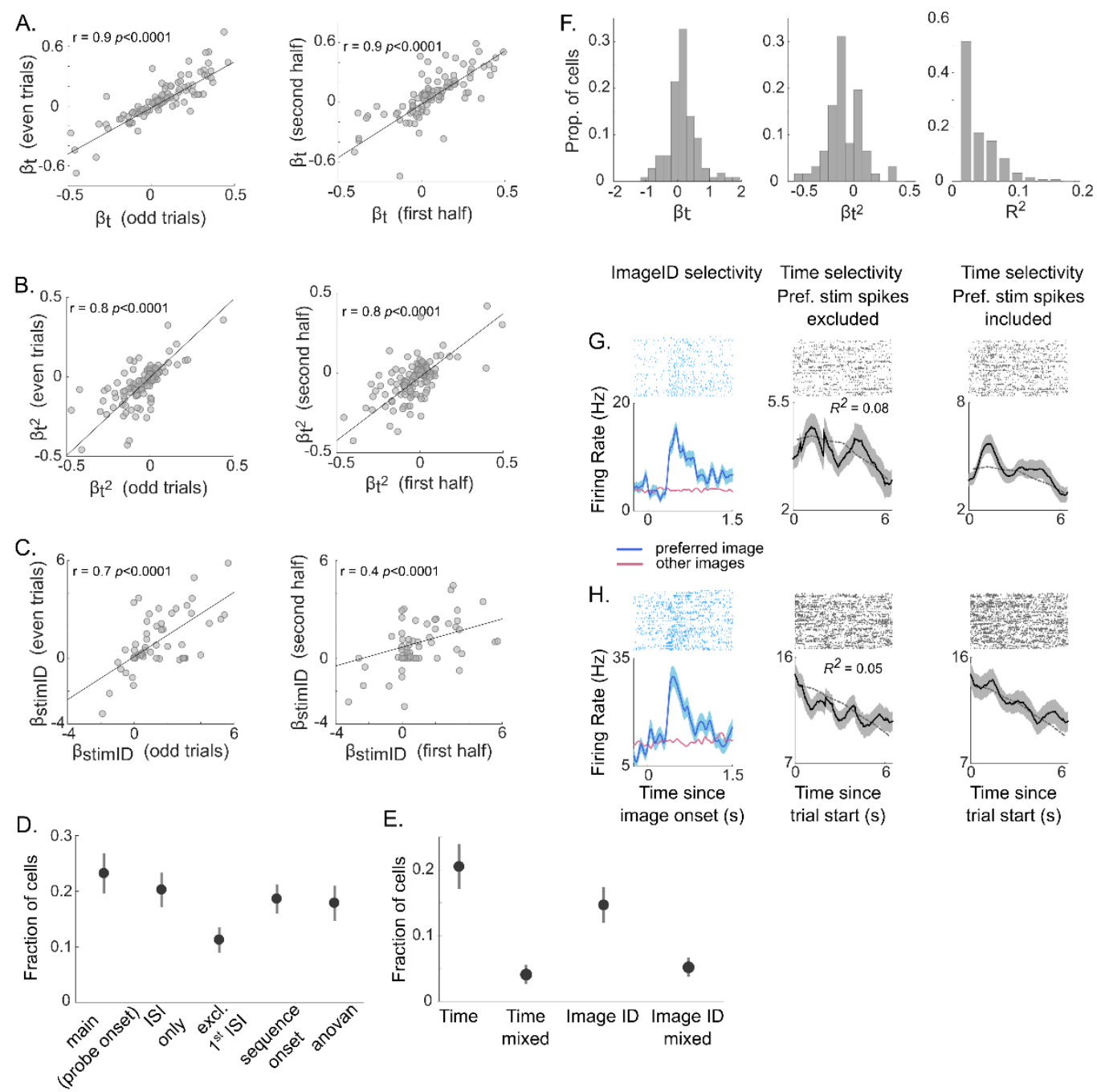

E.
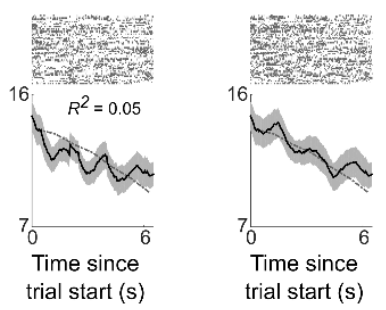

Figure 4. Stability of time cells (A, B) and image responsive cells $(C)$ across trials. The trials were split into odd/even trials, or first half/second half of trials. The beta coefficients for the time and time ${ }^{2}$ regressors are plotted for the time cells $(A, B)$, and for the imagelD regressor for the image responsive cells (C), in each subset of trials. D) Fraction of cells that were modulated by time in the main analysis (i.e., with respect to the offset of the probe event), when time cells were identified on only the ISI periods (ISI only), when the first ISI period was excluded 
(excl. $1^{\text {st }} \mathrm{ISI}$ ), when time cells were identified with respect to the onset of each iteration of the sequence (sequence onset), and with an N-way ANOVA. E) Fraction of cells that were exclusively modulated by time (Time), modulated 605 by time and another variable (Time mixed, e.g. Time and ImageID), exclusively modulated by image type (ImagelD), and modulated by image type and another variable (ImagelD mixed). The dots and vertical lines correspond to the mean and standard error of the mean across recording sessions respectively ( $N=31$ sessions). F) Distribution of beta coefficients for the linear and quadratic time terms, and $R^{2}$ values, for cells with significant time predictors. $G$, $H$ ). Examples of two cells that showed mixed selectivity for the factors of ImageID and time. left). These two cells

610 were modulated by the factor of ImageID. The raster plots and post-stimulus time histograms are aligned to the onset of each image in the sequence. The blue curve is the response to the image in the sequence that elicited the highest response (preferred image). The red curve is the mean response to the other images in the sequence. The blue shaded area is the standard error of the mean across trials of image presentation. right) The same two cells were also modulated by the factor of Time. The raster plots and post-stimulus time histograms are now aligned to

615 trial onset (i.e., the offset of the probe events). The format of the panels on the right is the same as Figure 3A. For visualization purposes only, and to better disentangle the effects of Time and ImagelD, the spikes corresponding to the preferred images have been excised from the middle panels. The gray lines show the model fit (these cells were classified as time cells based on the linear term).

\section{Internally generated time selectivity in human hippocampal neurons}

The results from Experiment 1 demonstrate that human hippocampal neurons are modulated by the temporal context of an explicit task. Do human hippocampal neurons also represent the temporal structure of an experience in the absence of external inputs or an overt task?

We performed a second experiment in six new patients who performed a different version of the sequence learning task (Figure 1B). In this new task, participants learned the sequence order as before, but every so often, the sequence stopped for 10 s and participants waited until 630 the sequence resumed. The participants had no stimulus input during these 10 sap periods -they were presented with a blank screen. We isolated 96 hippocampal neurons in this second experiment, and used a GLM approach to determine whether human hippocampal neurons represent temporal information during the gap periods. As before, significance testing was based on a permutation test in which we repeated the GLM $10^{6}$ times after shuffling the data 635 (Methods).

During the gap periods, 26 hippocampal neurons ( $27 \%$ of cells; Figure $5 \mathrm{~A}$ ) were significantly modulated by time (more than expected by chance based on a permutation test, $p<10^{-6}$ ), whereas while the patients were engaged in sequence-learning 13 neurons were time-selective.

640 Only 3 neurons encoded temporal information during both the task period and the gap period, suggesting that the recruitment of temporally sensitive cells can change with task demands or behavior (Pastalkova et al., 2008; MacDonald et al., 2011; Tsao et al., 2018; Umbach et al., 2020). During the gap periods, the time of peak firing in the population occurred at successive moments, and population activity spanned the entire 10s interval (Figure 5B). As above, the 645 reliability of the heat map was statistically verified with a cross-validation procedure (permutation test $p<10^{-5}$, Methods). As reported in rodents, there was a stronger representation of earlier timepoints (Salz et al., 2016). To summarize, even in the absence of 
visual input or an overt task, the firing activity of hippocampal neurons is inherently modulated by a changing temporal context.

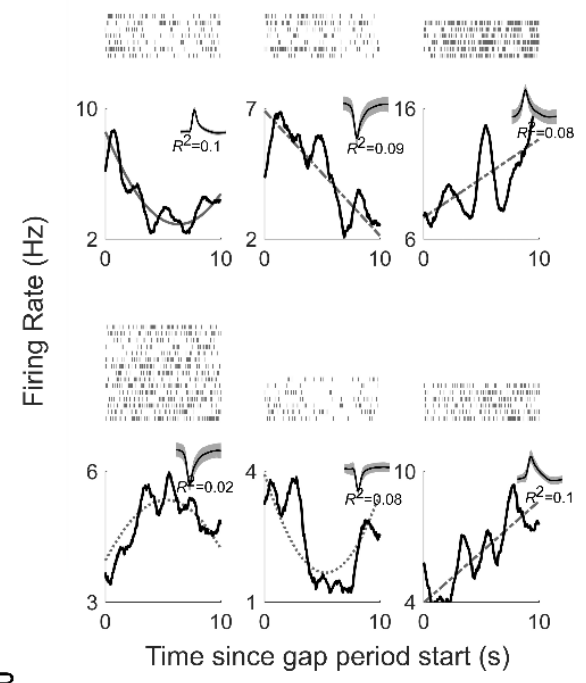

B

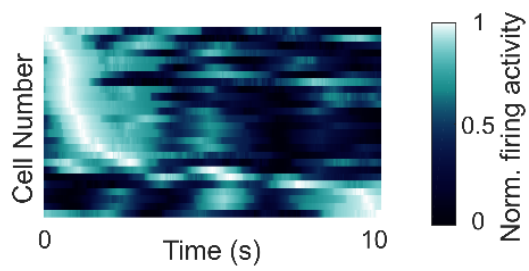

Figure 5. A) Hippocampal neurons are modulated by time during the gap periods. Raster plots (top) and poststimulus time histograms (bottom) are shown for 6 example time cells. The $x$-axis corresponds to the 10 s duration of the gap period. The gray lines show the model fit (solid gray line for cells that were classified as time cells according to the inclusion of the linear and quadratic terms, dashed gray line for cells classified based on the linear term alone, and stippled gray line for cells classified based on the quadratic term alone). The insets show the waveforms and the $R^{2}$ of the model for each cell. B) Hippocampal neurons fire at successive moments of the gap period. Firing activity of time cells in the temporal gap experiment $(N=26)$. Each row shows the firing activity for an individual cell, averaged across the gap periods. The $x$-axis corresponds to the 10 s gap period. The neurons are sorted by the latency of the maximum firing rate.

\section{Hippocampal population activity encodes temporal information.}

665 In the rodent brain, time information is signaled both explicitly in individual neurons, and can also be gleaned from population-level dynamics of time-selective and non-time-selective cells (Tsao et al., 2018). Is time information also reflected in the population activity of human hippocampal neurons? To address this question, we performed a population pattern analysis of image period identity in the sequence learning sessions (Figure 6). 
In Experiment 1, each trial of the experiment consisted of a sequence of image periods between two consecutive probes, and the goal of the population pattern analysis was to determine whether hippocampal population dynamics reflected the temporal identity of each image period (e.g., discriminate "image period 1" vs. "image period 2"). To ensure that 675 decoding or discrimination performance was not driven by an unequal representation of the different images in the different image periods, we created a balanced dataset in which accurate decoding of the temporal identity of each image period cannot arise from merely decoding spurious image information (Figure 6A). Decoding performance was based on a pairwise comparison method (Figure 6 B-D) as in (Haxby et al., 2001; Pereira et al., 2018); hence chance performance was $50 \%$ for all comparisons.

Discrimination performance was evaluated for different numbers of image periods from the start of the trial (ranging from two to five; i.e., discriminating between the first two image periods after the probe event, the first three image periods and so on). High decoding accuracy 685 for temporal period identity was observed for discriminating all image periods (Figure $6 \mathrm{E}$; mean \pm standard deviation of decoding accuracy for discrimination of the first two periods $=88.4 \pm 8 \%$, $\mathrm{t}(49)=34.8, \mathrm{p}<0.001 ;$ of the first three periods $=72.7 \pm 8 \%, \mathrm{t}(49)=20.1, \mathrm{p}<0.001$; of the first four periods $=63.5 \pm 6 \%, t(49)=14.9, p<0.001$; of the first five periods $=57.3 \pm 9 \%, t(49)=5.6, p$ $<0.001$ ). Decoding errors occurred primarily for neighboring periods (Figure 6F). Decoding 690 accuracy could not be biased by the offset of the probe events since the first ISI period after the probe event was excluded in the population pattern analysis. Thus, hippocampus population dynamics uniquely represented each temporal period.

To compare how temporal information is represented for time cells vs. non-time cells ("other 695 cells"), and to determine how population size affects decoding accuracy, we performed the pairwise pattern analysis separately for time cells and other cells, and for size-matched cell populations. As expected, decoding performance was consistently higher for time cells, but non-time cells also showed significant decoding suggesting that these cells encode temporal information, but possibly in a form that is not detected when modelled with a combination of linear and quadratic time terms, or only present in the population code (Figure 6, H).

Temporal epoch information was also present in population-level dynamics during the gap periods. A population pattern analysis during these gap periods revealed a significant representation of time information at the level of the overall population, and decoding errors 705 mainly occurred for neighboring epochs (Figure 6I, J). High decoding accuracy for temporal epoch identity was observed for different temporal epoch sizes (mean accuracy \pm standard deviation for 4 -way decoding $=76.0 \pm 4.7 \%, \mathrm{t}(49)=38.9, \mathrm{p}<0.001 ;$ for 5 -way decoding $=69.6 \pm$ $4.6 \%, \mathrm{t}(49)=29.9, \mathrm{p}<0.001$; and for 10 -way decoding $=65.4 \pm 3.1 \%, \mathrm{t}(49)=36.3, \mathrm{p}<0.001)$. Decoding accuracy during the gap periods could not have been driven by external visual input 710 or overt behavior; rather the high decoding accuracy reflects an internally generated temporal context signal represented in the population of neurons. 

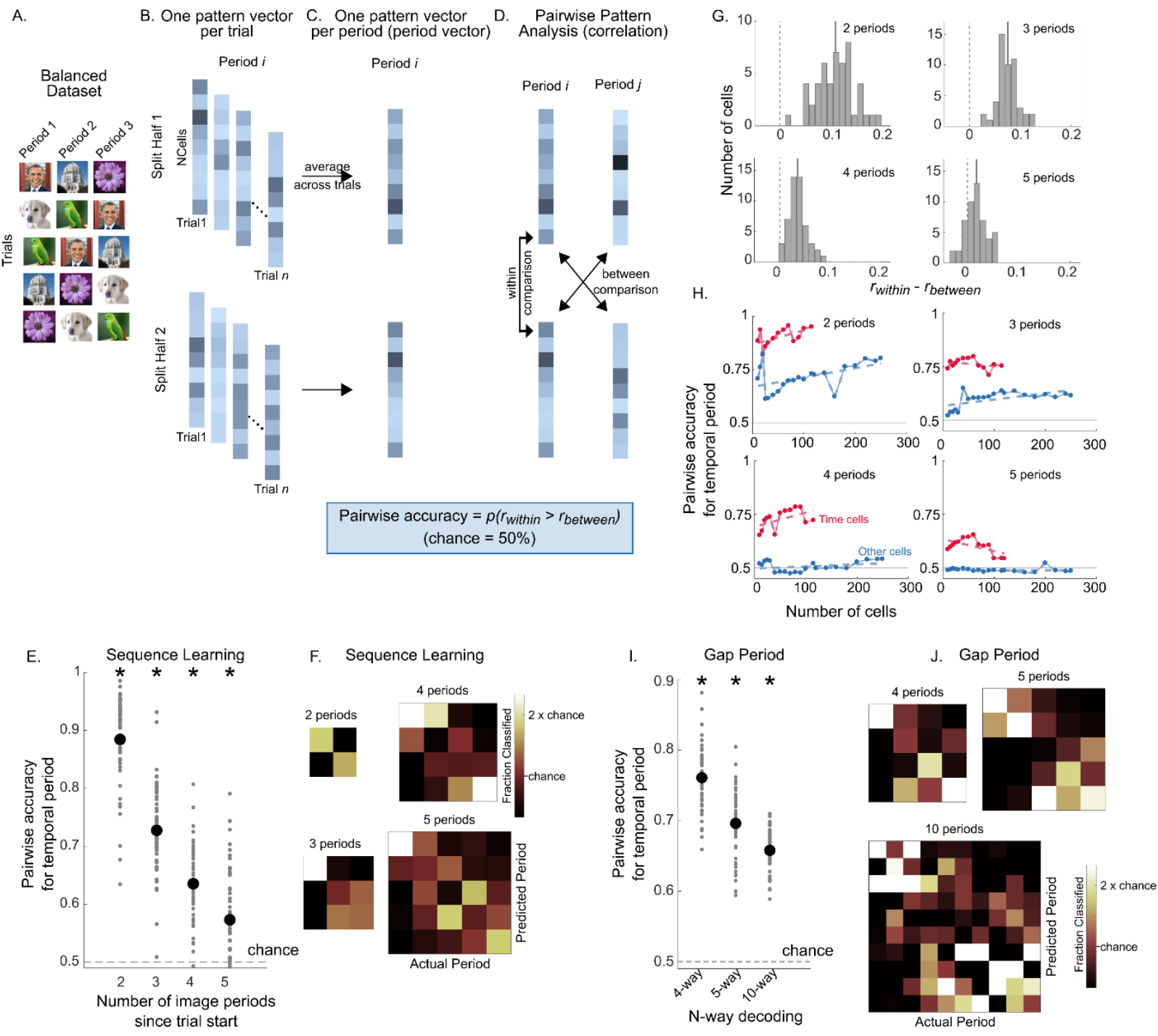

Figure 6: Population pattern analysis. In experiment 1, the population pattern analysis was performed on image periods (e.g., discriminating image period 1 vs image period 2). In experiment 2, the population pattern analysis was performed on the 10s gap interval. Since this decoding method was based on pairwise comparisons, chance performance is always $50 \%$. A). For experiment 1 , a balanced dataset was created by selecting a subset of trials so that each image was equally present in each period across trials (see methods). B) Population pattern analysis procedure. The trials of the balanced dataset (experiment 1), or the gap intervals (experiment 2) were split into two halves (repeated on 200 iterations; see methods). In each half of the dataset, and for each period, the firing activity of the population of cells was arranged into a pattern vector for each trial. C). An average pattern vector of population firing activity was obtained for each image period by averaging across trials (period vectors). D). Pairwise discrimination was performed on the period vectors across the two halves of the dataset. For all pairs of periods, the Pearson's correlation was computed across the two halves, and the same-period comparisons ("within comparisons") were compared to the different-period ("between") comparisons. Pairwise accuracy was the proportion of within comparison correlations $\left(r_{\text {within }}\right)$ that were higher than the between comparison correlations $\left(r_{\text {between }}\right)$ (Haxby et al., 2001; Pereira et al., 2018). E). Population pattern analysis accuracies. Pairwise accuracy for temporal period identity during the sequence learning experiment using the split-half procedure described in A-D (population size $=429$ neurons). The $x$-axis shows the number of image periods that the classifier was tested on (i.e., discrimination between the first two image periods, the first three image periods, etc., since the start of the trial). Pairwise accuracy (mean \pm standard deviation) for discriminating the first two periods $=88.4 \pm 8 \%$, of the first 
three periods $=72.7 \pm 8 \%$, of the first four periods $=63.5 \pm 6 \%$, of the first five periods $=57.3 \pm 9 \%$. The black dots correspond to the mean accuracy across the 50 iterations for creating the balanced dataset, and the gray dots show the distribution of accuracies obtained across iterations. Asterisks denote significance based on a t-test against chance, $\left(p<10^{-3}\right)$. Since the analysis is based on pairwise comparisons, chance performance is always $50 \%$. F) Decoding errors mainly occurred for predicting neighboring temporal periods. Confusion matrices during sequence learning when discriminating the first two temporal periods, the first three temporal periods etc. G).

740 Distributions of the difference of $\left(r_{\text {within }}\right)$ and $\left(r_{\text {between }}\right)$ for decoding of the first two image periods, the first three image periods etc. The solid gray line corresponds to the mean of the distribution. H). Pairwise accuracy for size matched populations of time cells (in red) and other (non-time) cells (in blue), for decoding of the first two image periods, the first three image periods etc. The dashed lines correspond to a linear fit through the data. The horizontal gray line corresponds to chance performance (50\%). I). Population pattern analysis performance during

745 the temporal gap experiment (population size=96 neurons). The 10s gap periods were split into four, five or ten discrete periods and discrimination of temporal period identity was computed using the procedure shown in B-D. Pairwise accuracy was significantly above chance for all comparisons (t-test against chance $(0.5), p<10^{-3}$ ). Pairwise accuracy, mean \pm standard deviation $=76.0 \pm 4.7 \%$ for 4 -way decoding, $69.6 \pm 4.6 \%$ for 5 -way decoding, and $65.7 \pm$ $3.1 \%$ for 10 -way decoding. The black dots correspond to the mean accuracy across the 50 iterations for creating the balanced dataset, and the gray dots show the distribution of accuracies obtained across iterations. Asterisks denote significance based on a t-test against chance, $\left(p<10^{-3}\right)$. Since the analysis is based on pairwise comparisons, chance performance is always 50\%. J) Confusion matrix for the gap experiment for different epoch lengths.

\section{Discussion:}

In this study we report that human hippocampal neurons represent temporal information as subjects progress through a sequence of events, and during empty gap periods in the sequence. Time cells responded successively at different moments of the task, and together, the activity 760 of these neurons covered the entire task period. Population level activity allowed for successful decoding of temporal epoch identity.

Temporal information is represented at different time scales in the hippocampus and neighboring brain regions. In rodents, hippocampal time cells typically show sharp tuning for

765 particular moments of a fixed interval (Pastalkova et al., 2008; MacDonald et al., 2011), whereas in the lateral entorhinal cortex, temporally sensitive neurons show a more gradual ramping of firing activity (Tsao et al., 2018). In humans, population activity gradually drifts over a period of minutes (Folkerts et al., 2018), but single neurons can also show punctuated timecell-like firing patterns as we show here and as previously reported (Umbach et al., 2020). The

770 relationship between a gradually changing temporal context versus a more precise representation of time in the human hippocampus remains an open question for future work. Task demands such as free recall versus precise temporal order judgments, and the resolution at which temporal information is relevant at the behavioral level may influence the temporal precision of human time cells.

775

Rodent time cells display flexibility in temporal coding. For instance, they exhibit scalar coding of time, such that cells that are active later in a time window fire for longer periods. Further, just like place cells re-map, time cells have been observed to "re-time": cells can change their temporal preferences within the same recording session when the temporal structure of the 780 experience is changed (MacDonald et al., 2011). For our patients, the image sequence was 
generally fixed across sessions and patients typically performed only one experimental session per day. We were thus unable to test whether temporal preferences change when the image order of the sequence changes or when the same sequence is repeated after a short interval. Observations of re-timing in rodent time cells raise intriguing possibilities for future investigations of temporal coding in the human hippocampus.

Perhaps most relevant to the current study are recent findings of Umbach and colleagues (Umbach et al., 2020). That study reported time cell activity in the human medial temporal lobe during a free recall memory task. Hippocampal neurons encoded temporal information during 790 the task, and entorhinal cortex neurons showed ramping activity. Unlike our task design which consisted of a structured and predictable sequence of items that repeated continually for 10-15 minutes, the free recall task in Umbach et al., consisted of two distinct phases, one in which words were presented in a structured list, and another that consisted of a period of free recall. Both studies report a significant number of time cells, although we report a higher proportion 795 (30\%) compared to the Umbach et al., study (15\%). This difference cannot solely be due to the different methodology used in the two studies for detecting time cells (stepwise regression vs. a one-way ANOVA), since we identified $\sim 24 \%$ of time cells when using an ANOVA as our model. Instead, it seems likely that the different proportions of time cells could arise from differences in task structure or behavioral requirements. For instance, the hippocampus is essential for 800 remembering the temporal order of sequential events (Eichenbaum, 2013), and it is possible that temporal order learning engages a larger population of cells for representing time information. The study by Umbach and colleagues also showed that time cell activity was relevant to subsequent performance on a memory test. Our study did not directly test for the relationship between time cell firing and behavioral performance since the task was expressly 805 designed to be easy for patients to perform. As such, we did not have enough error trials to correlate time cell activity with performance on the sequence memory task. Nevertheless, despite these differences in task design and methodology, both the study by Umbach et al., and our study report a significant proportion of hippocampal neurons that are modulated by time, suggesting that a temporal code may be a general property of the human hippocampus 810 network.

The temporal modulation of time cells in our study was also observed during empty 10s gap periods in which patients were not presented with any visual input or required to perform an explicit task. Time cells were observed to fire at successive moments in these blank periods.

815 Temporal modulation during these gap periods could not have been driven by external events; rather they appear to represent an evolving temporal signal as a result of changes in the patients' experience during this time of waiting. Related to this point, temporal coding in the hippocampus and the lateral entorhinal cortex has been shown to change with behavior and task demands (MacDonald et al., 2013; Tsao et al., 2018). The design of Experiment 2 allowed

820 us to directly examine whether cells that were modulated by time during sequence learning were also recruited during the gap periods when the patients did not overtly perform a task. We found that different subsets of cells were active during these two task periods, with a small overlap of cells that were active in both periods. These results suggest a context-dependent recruitment of cells for the representation of temporal information, and raise interesting 
825 questions for future research of how the encoding of temporal information in single cells and at the population level is affected when switching between tasks or experiences.

We found evidence for time modulation in various control analyses that considered the trial structure (i.e., sequence time vs. probe time), trial duration (trials of a fixed duration (median length) vs. all trials), or trial contents (e.g., with or without image information). Interestingly,

830 we found that excluding the first ISI period of each trial from the analysis resulted in an important difference in the number of time cells identified (Table 1). This finding, along with the heatmaps shown in Figures $3 B$ and 5B, suggests that a large number of cells show firing rate changes shortly after the probe, similar to reports in rodents and humans of a stronger representation of temporal information early in the trial (Salz et al., 2016; Umbach et al., 2020).

835 Importantly however, this early representation of temporal information did not compromise population-level representations of elapsed time since our decoding analyses, performed on image periods only, showed robust temporal information at the population level.

In rodents, time cells and place cells do not uniquely represent temporal and spatial 840 information respectively. Rather, medial temporal lobe neurons can be influenced by various experimental factors, including the stimulus-related, spatial and temporal facets of an experience (Komorowski et al., 2009; Tsao et al., 2018). For example, in rodents it has been reported that place cells can code for distance, time, or visual cues (Ravassard et al., 2013; Eichenbaum, 2014; Acharya et al., 2016), and time cells can encode spatial or stimulus 845 information (Eichenbaum, 2014; Tsao et al., 2018). Similarly, we found that human time cells also encoded sensory information about the presence or absence of a stimulus, and the identity of the stimulus. Such multi-dimensional representations could play a critical role in episodic memory mechanisms in which the "what", "where", and "when" elements of an experience are bound together into a coherent memory.

The phenomenon of subjective "mental time travel" is a cornerstone of episodic memory (Tulving, 2002). Central to our experience of reliving the past is our ability to vividly recall specific events that occurred at a specific place and in a specific temporal order. Time cells in rodents and humans, and other temporally-sensitive populations of neurons support 855 theoretical frameworks that posit that temporal context information plays an important role in memory mechanisms in the hippocampus (Howard et al., 2014; Howard et al., 2015). Our results provide further evidence that human hippocampal neurons represent the flow of time in an experience. 


\section{References:}

Acharya L, Aghajan ZM, Vuong C, Moore JJ, Mehta MR (2016) Causal Influence of Visual Cues on Hippocampal Directional Selectivity. Cell 164:197-207.

865 Eichenbaum H (2013) Memory on time. Trends in cognitive sciences 17:81-88.

Eichenbaum H (2014) Time cells in the hippocampus: a new dimension for mapping memories. Nature reviews Neuroscience 15:732-744.

Ekstrom AD, Bookheimer SY (2007) Spatial and temporal episodic memory retrieval recruit dissociable functional networks in the human brain. Learning \& memory 14:645-654.

870 Engel AK, Moll CK, Fried I, Ojemann GA (2005) Invasive recordings from the human brain: clinical insights and beyond. Nature reviews Neuroscience 6:35-47.

Folkerts S, Rutishauser U, Howard MW (2018) Human Episodic Memory Retrieval Is Accompanied by a Neural Contiguity Effect. The Journal of neuroscience : the official journal of the Society for Neuroscience 38:4200-4211.

875 Fortin NJ, Agster KL, Eichenbaum HB (2002) Critical role of the hippocampus in memory for sequences of events. Nature neuroscience 5:458-462.

Fried I, MacDonald KA, Wilson CL (1997) Single neuron activity in human hippocampus and amygdala during recognition of faces and objects. Neuron 18:753-765.

Gelbard-Sagiv H, Mukamel R, Harel M, Malach R, Fried I (2008) Internally generated reactivation

880 of single neurons in human hippocampus during free recall. Science 322:96-101.

Hasselmo ME (2009) A model of episodic memory: mental time travel along encoded trajectories using grid cells. Neurobiol Learn Mem 92:559-573.

Haxby JV, Gobbini MI, Furey ML, Ishai A, Schouten JL, Pietrini P (2001) Distributed and overlapping representations of faces and objects in ventral temporal cortex. Science 885 293:2425-2430.

Haxby JV, Guntupalli JS, Connolly AC, Halchenko YO, Conroy BR, Gobbini MI, Hanke M, Ramadge PJ (2011) A common, high-dimensional model of the representational space in human ventral temporal cortex. Neuron 72:404-416.

Howard MW, Viskontas IV, Shankar KH, Fried I (2012) Ensembles of human MTL neurons "jump 890 back in time" in response to a repeated stimulus. Hippocampus 22:1833-1847.

Howard MW, Shankar KH, Aue WR, Criss AH (2015) A distributed representation of internal time. Psychological review 122:24-53.

Howard MW, MacDonald CJ, Tiganj Z, Shankar KH, Du Q, Hasselmo ME, Eichenbaum H (2014) A unified mathematical framework for coding time, space, and sequences in the hippocampal region. The Journal of neuroscience : the official journal of the Society for Neuroscience 34:4692-4707.

Komorowski RW, Manns JR, Eichenbaum H (2009) Robust conjunctive item-place coding by hippocampal neurons parallels learning what happens where. The Journal of neuroscience : the official journal of the Society for Neuroscience 29:9918-9929.

900 Kraus BJ, Robinson RJ, 2nd, White JA, Eichenbaum H, Hasselmo ME (2013) Hippocampal "time cells": time versus path integration. Neuron 78:1090-1101. 
Kraus BJ, Brandon MP, Robinson RJ, 2nd, Connerney MA, Hasselmo ME, Eichenbaum H (2015) During Running in Place, Grid Cells Integrate Elapsed Time and Distance Run. Neuron 88:578-589.

905 MacDonald CJ, Lepage KQ, Eden UT, Eichenbaum H (2011) Hippocampal "time cells" bridge the gap in memory for discontiguous events. Neuron 71:737-749.

MacDonald CJ, Carrow S, Place R, Eichenbaum H (2013) Distinct hippocampal time cell sequences represent odor memories in immobilized rats. The Journal of neuroscience : the official journal of the Society for Neuroscience 33:14607-14616.

910 Manns JR, Howard MW, Eichenbaum H (2007) Gradual changes in hippocampal activity support remembering the order of events. Neuron 56:530-540.

Park IM, Meister ML, Huk AC, Pillow JW (2014) Encoding and decoding in parietal cortex during sensorimotor decision-making. Nature neuroscience 17:1395-1403.

Pastalkova E, Itskov V, Amarasingham A, Buzsaki G (2008) Internally generated cell assembly 915 sequences in the rat hippocampus. Science 321:1322-1327.

Pereira F, Lou B, Pritchett B, Ritter S, Gershman SJ, Kanwisher N, Botvinick M, Fedorenko E (2018) Toward a universal decoder of linguistic meaning from brain activation. Nature communications 9:963.

Quiroga RQ, Nadasdy Z, Ben-Shaul Y (2004) Unsupervised spike detection and sorting with

wavelets and superparamagnetic clustering. Neural computation 16:1661-1687.
Ravassard P, Kees A, Willers B, Ho D, Aharoni DA, Cushman J, Aghajan ZM, Mehta MR (2013) Multisensory control of hippocampal spatiotemporal selectivity. Science 340:1342-1346.

Reddy L, Poncet M, Self MW, Peters JC, Douw L, van Dellen E, Claus S, Reijneveld JC, Baayen JC, Roelfsema PR (2015) Learning of anticipatory responses in single neurons of the human medial temporal lobe. Nature communications 6:8556.

Salz DM, Tiganj Z, Khasnabish S, Kohley A, Sheehan D, Howard MW, Eichenbaum H (2016) Time Cells in Hippocampal Area CA3. The Journal of neuroscience : the official journal of the Society for Neuroscience 36:7476-7484.

Spiers HJ, Burgess N, Hartley T, Vargha-Khadem F, O'Keefe J (2001) Bilateral hippocampal pathology impairs topographical and episodic memory but not visual pattern matching. Hippocampus 11:715-725.

Tsao A, Sugar J, Lu L, Wang C, Knierim JJ, Moser MB, Moser El (2018) Integrating time from experience in the lateral entorhinal cortex. Nature 561:57-62.

Tulving E (2002) Episodic memory: from mind to brain. Annual review of psychology 53:1-25.

935 Umbach G, Kantak P, Jacobs J, Kahana M, Pfeiffer BE, Sperling M, Lega B (2020) Time cells in the human hippocampus and entorhinal cortex support episodic memory. Proceedings of the National Academy of Sciences of the United States of America 117:28463-28474. 